\title{
Meiotic chromosome behaviour and karyomorphology of Aloe vera (L.) Burm. f.
}

\author{
Ajey Karan Chaudhari and B. R. Chaudhary ${ }^{1}$ \\ Laboratory of Cytogenetics, Department of Botany, \\ Banaras Hindu University, Varanasi-221005, India \\ 'Author for correspondence: (brchaudhary.bhu@gmail.com) \\ Received February 9, 2012; accepted February 29, 2012
}

\begin{abstract}
Aloe vera (L.) Burm. f. (the family Aloaceae) indigenous to Africa is an admired medicinal plant used as a folk medicine. Meiotic division was investigated in A. vera with a view to decipher the behavior of chromosomes during prophase I and the subsequent stages of meiosis following acetocarmine squash technique. The various meiotic stages from leptotene to pollen development were studied. Seven bivalents were clearly visible at diplotene, and the chiasma frequency averaged to 1.86 per bivalent and 13.1 per pollen mother cell (PMC). Prophase I stages are difficult to identify due to clumping of chromosome threads. Second nuclear division, however, allows clear distinction of chromosome configuration. Besides clumping, various other types of chromosomal aberrations such as stickiness and fragments at metaphase I, precocious movement of chromosomes, bridges, laggards and unequal separation of chromosomes at anaphase I and unequal separation of chromosomes at telophase II were noted. Karyomorphological study on the other hand, was carried out in anther wall (mitotic) cells of $A$. vera. Mitotic as well as meiotic studies in the species showed $2 \mathrm{n}=14$ chromosomes including two pairs of sat-chromosomes. The karyotype of the species was adjudged to be typically asymmetric.
\end{abstract}

KEYWORDS: Aloe vera, Chromosome anomalies, Karyomorphology, Meiosis

Aloe belongs to the family Aloaceae consisting of perennial tropical plants of African origin. More than 360 species are known worldwide in the warm regions of Africa, Europe, Asia and the Americas. Records of the use of Aloe vera (L.) Burm. f. as folk medicine date back to antiquity with an early account from around 1500 B.C. Of the four species found in India, Aloe vera is most widely naturalized. Basically, all the species of Aloe have similar constituents, but $A$. vera is more popular all over the world because it propagates faster than any other known species of Aloe (Anselm 2004). Aloe vera is small, stemless, herbaceous and perennial plant with shallow root system. The fleshy, sword-shaped leaves are gray-green and grow to $80-90 \mathrm{~cm}$ long. Younger leaves have pale spots. The plant slowly offsets to form a clump. Only large plants flower in November-December. The unbranched flower spike carries tubular yellow flowers.

Scientific investigations on $A$. vera led to believe that it is effective in the treatment of stomach ailments, skin diseases, radiation injury (Fine and Samuel 1938; Grindlay and Reynolds 1986; Shelton 1991), wound healing (Heggers et al. 1993) and cancer (Winters et al. 1981).

In recent years, Aloe species have attracted the attention of geneticists and cell biologists with respect meiotic process and meiosis-driven genome variation in view of the increasingly greater genetic/ genomic variability encountered in this plant. The medicinal value and male sterility of $A$. vera make it important for cytogenetical investigations. Aloe vera is predominantly characterized by the $2 \mathrm{n}=2 \mathrm{x}=14$ chromosomes with four long and three small chromosome in its basic haploid set (Vig 1968). Despite long history of study and the recent rapid progress in biochemical study, cytological investigation of $A$. vera has progressed slowly and unsteadily because of the extremely abnormal features of meiosis and occasional flowering compared to most other angiosperms. Yet, its importance is partly associated with general interest in plant reproduction and fertility and partly with greater understanding of genome constitution and its exploitation. In contrast to spontaneous mitotic chromosomal aberrations (Vig 1968; Sapre 1975), somatic chromosome characteristics (Sapre 1978; Umesh and Ranganath 2003; Das et al. 2010), including the fluorescent metaphase chromosomes (Alam and Khanam 2005) reported earlier throwing light on the structure and behaviour of mitotic chromosomes, meiotic behaviour especially of prophase I chromosomes is inadequate. The present study aims at providing a comprehensive account of the structure and behaviour of meiotic as well as mitotic chromosomes of $A$. vera.

\section{Materials and Methods}

The plants used in this study consisted of the wild type of Aloe vera maintained in the Botanical Garden, Banaras Hindu University, Varanasi, India. Flower buds of appropriate sizes were collected from plants and fixed in Carnoy's fluid (3 ethanol: 1 acetic acid; v/v) for $24 \mathrm{~h}$, and then, they were transferred to $70 \%$ alcohol and stored at $4{ }^{\circ} \mathrm{C}$. Acetocarmine solution at concentration of $1 \%$ was prepared by dissolving $1 \mathrm{~g}$ of synthetic carmine dye in $45 \%$ acetic acid ( $45 \mathrm{ml}$ of acetic acid and $55 \mathrm{ml}$ of distilled water) in a condenser fitted conical flask over a flame of spirit lamp for 4-5 hrs. The solution was cooled down, filtered and stored in a dark bottle at $4^{\circ} \mathrm{C}$ for its use.

Flower buds varying in size $(0.5-1.5 \mathrm{~mm})$ and arranged 
in ascending order in a watch glass/petridish containing fixative were transferred individually on to a clean glass slide, and a single anther was dug up from flower bud. A small drop of $1 \%$ acetocarmine stain was placed over the anther and the latter was gently tapped with flat spatula to release pollen mother cells into the stain. The anther lobe wall/ debris was carefully removed from the stain before placing on to it the coverslip. The slide was then gently warmed to improve staining of the chromosomes. The preparation was then gently squashed using vertical thumb pressure applied on to the coverslip under double fold of filter paper. Well stained and squashed cells were examined for the structure and behaviour of chromosomes during prophase and the subsequent stages of meiotic division, and the appropriate stages were photographed using a Catalyst Biotech Cat Cam DCM300 Digital Camera. Images of analyzed cells were processed with Adobe Photoshop 7.0 software to enhance contrast of the chromosome threads at early stages of meiotic division.

Approximately 250 PMCs from arbitrarily selected microscopic fields were examined and the abnormalities noticed in different phases of meiosis were recorded. Karyotype study was done from the squashed up anther lobe wall cells which divide mitotically. Ten metaphase nuclei, with properly stained and separated chromosomes were evaluated to record the following chromosomal features characterizing the karyotype; (i) Number of chromosomes (ii) Absolute size of chromosomes, (iii) arm ratio, L/S (iv) haploid chromosome length, (v) mean chromosome length, and (vi) ratio of longest to shortest chromosome following Levan et al. (1964). The nature of karyotype was assessed using ratio of the longest to the shortest chromosomes and the proportion of chromosomes with arm ratios as proposed by Stebbins (1971). Pollen stainability test was performed on open flowers to test pollen sterility. About one thousand pollen grains were examined for the percentage of viable and non-viable pollen grains. The filled-up, spherical/hemispherical and deeply stained pollen grains were counted as viable, while weakly stained, off-size and mis-shaped pollen grains were regarded as non-viable ones (Singh and Dhuria 1960).

\footnotetext{
Results

Investigations on meiotic chromosomes In earlier studies, meiotic prophase was not properly described because of the extremely sticky nature of the chromosomes (Sapre 1975). This difficulty was overcome mainly through preparation of a large number of slides and rigourous selection for each of the division sub-stages. In early prophase I, leptotene nuclei are abundant, and have quite tenuous chromosome threads with heavily stained and interspersed regions presumed to be the large heterochromatin blocks (Fig. 1A). Pairing between homologous chromosomes in the zygotene stage (Fig. 1B) is not easy to decipher because the process of synapsis occurs when
}

the chromosomes are highly extended, tenuous and intertwined. Occasionally, boundaries between synapsed and unsynapsed regions can be identified as forked structures in the late zygotene nuclei. Synapsed regions apper as thick segments reflecting close apposition of the homologous chromosomes, which distinguish them from unsynapsed thin chromosome regions. Pachytene chromosomes become more condensed vis a vis conspicuous exhibiting beaded appearance (Fig. 1C). The diplotene stage (Figs. 1D and 1E) exhibits still more condensed and darkly stained thick chromosomes showing seven bivalents with variable chiasmata. At diplotene, while chiasma frequency (average of 10 PMCs) ranged from 15 to11 chiasmata/PMC, the frequency of chiasma averaged to 1.86 per bivalent and 13.1 per PMC. In $A$. vera, due to heavy degree of chromosome clumping at diplotene and diakinesis most of the preparations of these ideal stages do not exhibit well separated bivalents and the chiasmatic regions. In well spread preparations at diakinesis stage (Fig. 1F) seven bivalents comprising four long subtelocentric and three comparatively very small submetacentric chromosome pairs with terminalized chiasmata were encountered. At metaphase I, the seven heavily condensed and fully heterochromatized bivalents arranged at the equator may even be counted in good squash preparations (Fig. 1G). At anaphase I (Fig. 1H) homologous chromosomes each consisting of two chromatids, migrate to the two poles following the abrupt cessation of sister chromatid cohesion. At early telophase I (Fig. 1I) the chromosomes that have reached to the poles become aggregated to loose their individuality and appear as clumped chromosome mass.

The chromosomes continue to condense and reach maximum degree of contraction at metaphase II. At anaphase II (Fig. 1J) individual chromatids separate and migrate to the spindle poles perpendicular to the plane of of 1st nuclear division resulting into four groups of chromatids in a meiocyte (Fig. 1K), which gradually transform into tetrads each containing haploid number of chromosomes (Fig. 1L). Eventually, the cytoplasm becomes partitioned by cytokinesis and the four haploid microspores continue their development into mature pollen grains. The darkly stained spherical or hemispherical pollen grains accounted for $87.60 \%$ viable pollens in $A$. vera.

Spontaneous meiotic chromosome anomalies The stages at which chromosome aberrations were encountered are metaphase I, anaphase I, telophase I, and all the subsequent stages of the second nuclear division. The data on various aberrations such as stickiness, bridges, fragments, precocious movement, unequal separation and laggards were presented in Table 1. In A. vera, meiotic chromosomes showed frequent occurrence of stickiness and bridges of chromosomes at metaphase I and anaphase I, respectively (Figs. 2A and 2D). The bridges single or double were the 

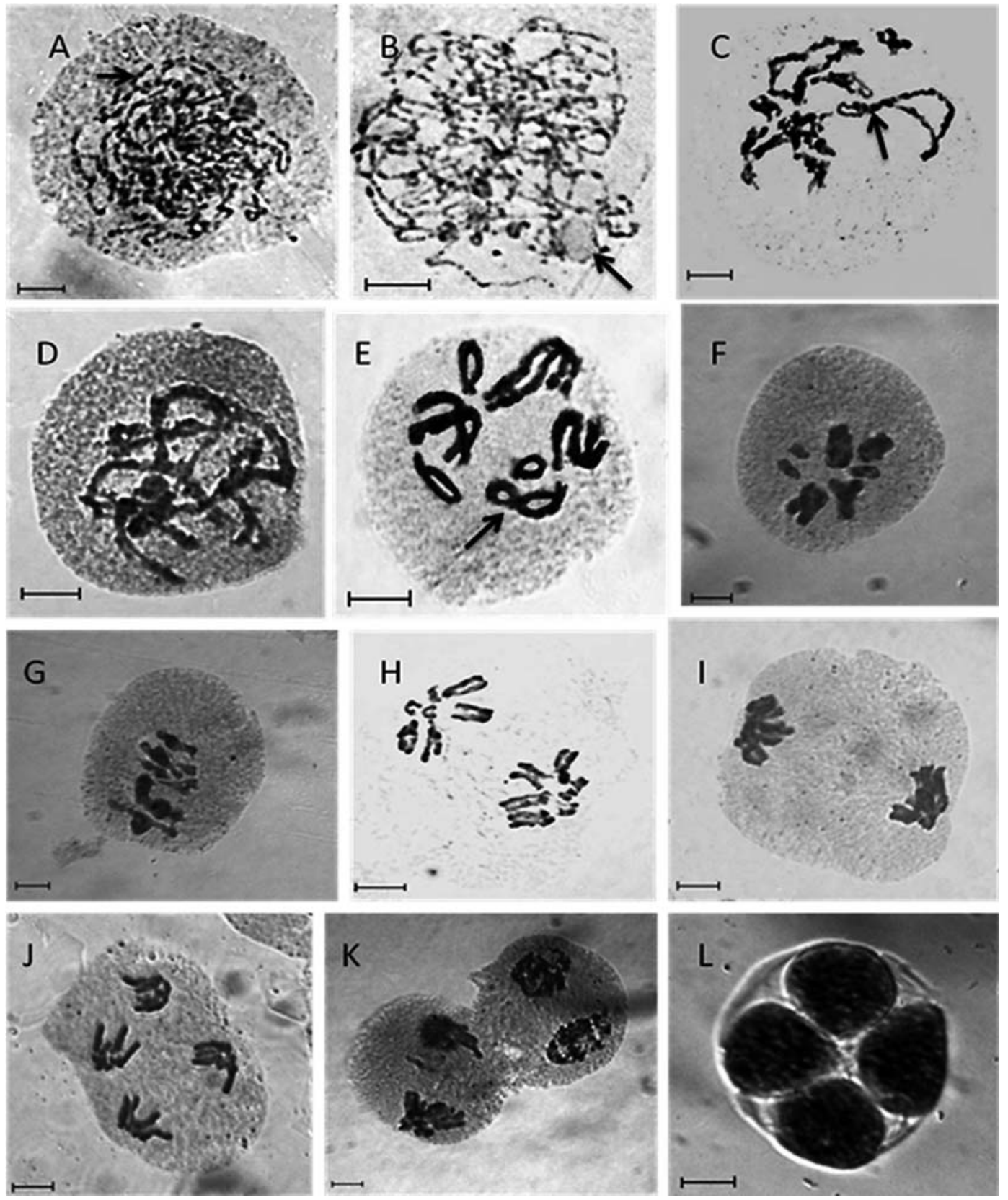

Fig.1. Meiotic stages from acetocarmine-stained squash preparations of pollen mother cells of Aloe vera. A. Leptotene, showing unsynapsed chromatin threads; arrow indicates heterochromatin; B. Zygotene, showing well dispersed and conspicuous chromatin threads; arrow indicates nucleolus; C. Pachytene, showing thick and beaded chromosomes; D. Diffuse diplotene with thick chromosomes; E. Late diplotene, showing seven bivalents with chiasma; F. Diakinesis, showing four long and three short bivalents; G. Metaphase I showing seven bivalents arranged at the equator; H. Anaphase I, showing chromosomes with distinct sister chromatids migrated to the poles; I. Late anaphase I, chromosomes have moved to the poles; J. Anaphase II, showing four groups of chromatids two in each diad cells; K. Showing two groups of chromatin in each diad cells; L. Four pollen grains formed in a meiocyte. $(\mathrm{Bar}=10 \mu \mathrm{m})$

Table 1. Percentage of spontaneous chromosome aberrations encountered in Aloe vera

\begin{tabular}{cccccccccc}
\hline \multirow{2}{*}{$\begin{array}{c}\text { No. of } \\
\text { dividing } \\
\text { cells }\end{array}$} & \begin{tabular}{c} 
No. of \\
abnormal \\
\cline { 5 - 10 }
\end{tabular} & cells & Stickiness & $\begin{array}{c}\text { Single } \\
\text { bridges }\end{array}$ & $\begin{array}{c}\text { Double } \\
\text { bridges }\end{array}$ & Fragments & $\begin{array}{c}\text { Precocious } \\
\text { movement }\end{array}$ & $\begin{array}{c}\text { Unequal } \\
\text { separation }\end{array}$ & $\begin{array}{c}\text { Laggards } \\
\text { \% chromosome } \\
\text { aberration }\end{array}$ \\
\hline 250 & 41 & $4.4(11)$ & $4.8(12)$ & $3.2(8)$ & $0.4(1)$ & $0.8(2)$ & $(1.6) 4$ & $(1.2) 3$ & 16.4 \\
\hline
\end{tabular}

Values in parenthesis represent the number of cells 
most common; single bridges (Fig. 2D) were maximum (4.8\%) while fragments (Fig. 2B) were the minimum $(0.4 \%)$ amongst all the kinds of aberrations noted. The frequency of chromosomal aberrations (i.e. 16.4\%) was accounted by $4.41 \%$ stickiness (Fig. $2 \mathrm{~A}$ ), $0.8 \%$ precocious movement (Fig. 2C), 3.2\% double bridges (Fig. 2E), 1.2\% laggards (Fig. 2F) and 1.6\% unequal separation (Figs. 2GI), indicating thereby a high degree of meiotic irregularity and pollen inviability in natural plant population of $A$. vera.

Karyotype study in somatic/mitotic anther wall cells The anther wall cells are somatic and show mitotic division. Well spread metaphase plates in the vegetative cells of anther lobes showed $2 n=14$ chromosomes with eight long subtelocentric and six short submetacentric chromosomes (Figs. 3A-B). The length of chromosomes ranged from 5.75 to $16.60 \mu \mathrm{m}$ in size. Aloe vera presented $77.85 \mu \mathrm{m}$ as total chromosome length of the haploid set and $11.12 \mu \mathrm{m}$ as the average length of chromosome. Ratio of the largest to the smallest chromosomes was found to be 2.88 with $26.98 \%$ centromeric index. Long chromosome pairs I and IV having secondary constriction at long arm of the chromosomes have been represented in the idiogram (Fig. 4). Based on karyotypic features $A$. vera has been classed in the 3B classification of Stebbins (1971), which corresponds to asymmetric karyotype. The karyotype formula (KF) $4 \mathrm{St}^{\text {sat }}+4 \mathrm{St}+6 \mathrm{Sm}$ has been presented following the chromosome nomenclature given by Levan et al. (1964) on the basis of centromeric position.

\section{Discussion}

Meiotic chromosome analysis The purpose of presenting this atlas of meiosis and chromosomal aberrations in the wild type of Aloe vera is to provide basic outline of meiosis vis-a-vis investigation of somatic chromosomes in anther wall cells to support the earlier data of karyotype study. This outline description would serve as a useful
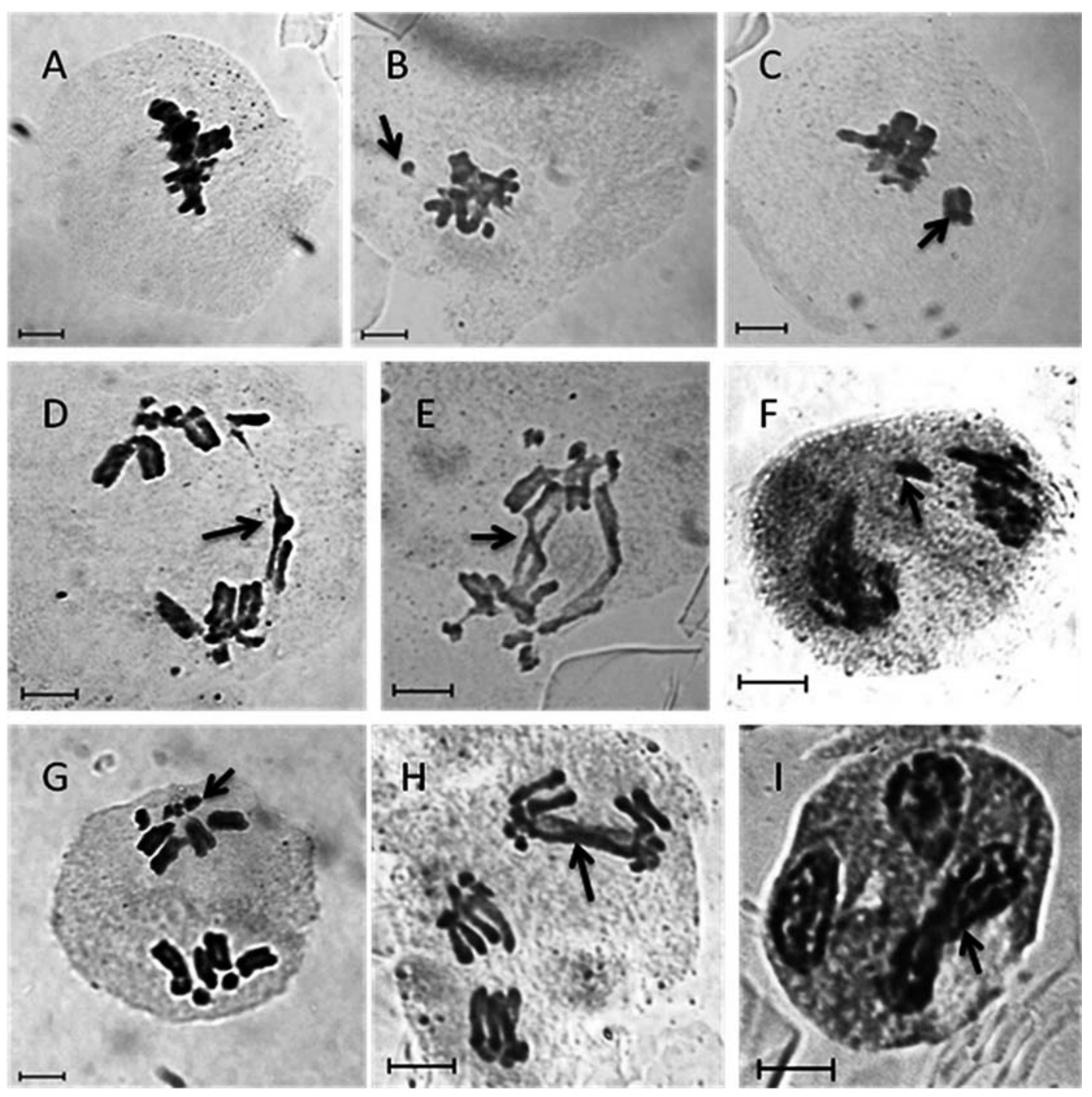

Fig. 2. PMCs showing meiotic chromosomal aberrations in Aloe vera. A. Stickiness at metaphase I; B. Fragments at metaphase I; C. Precocious movement of chromosomes at early anaphase I; D. Single bridge at anaphase I; E. Double bridge at anaphase I; F. Laggards at anaphase I; G. Unequal separation of chromosome at anaphase I; H. Unequal separation of chromosome at anaphase II; I. Unequal separation of nuclei at telophase II. (Bar $=10 \mu \mathrm{m})$ 


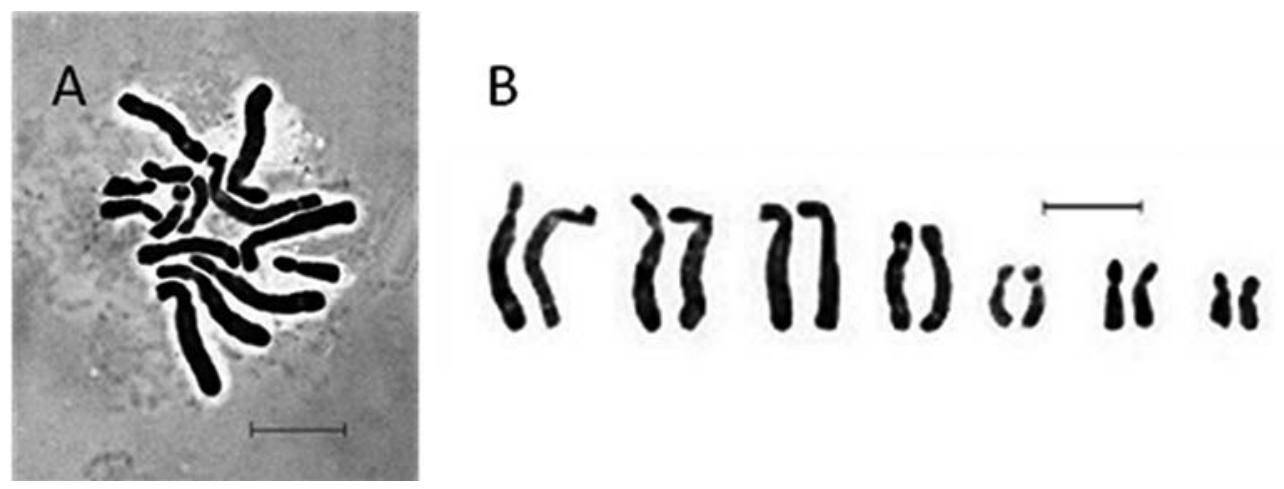

Fig. 3. A. Acetocarmine stained metaphase chromosomes of Aloe vera showing $2 n=14$ chromosomes; B. Karyogram showing four long subtelocentric pairs and the three short submetacentric pairs (Bar $10 \mu \mathrm{m})$

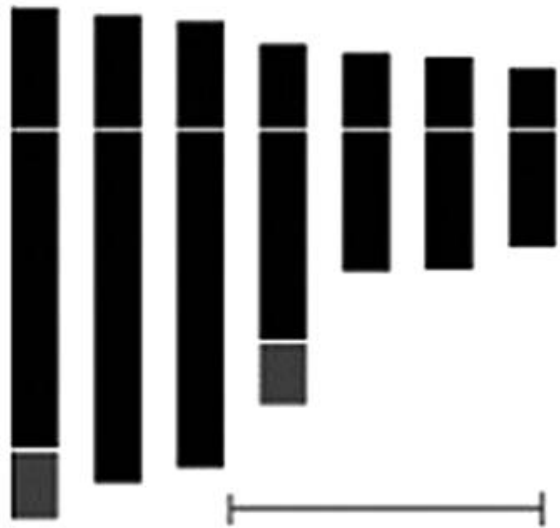

Fig. 4. Idiogram of Aloe vera. (Bar=10 $\mu \mathrm{m})$

framework for meiotic investigations through acetocarmine staining procedure, which is inexpensive, quick and adequate for visualizing chromosomes in meiotic squash preparations. As expected, early prophase I analysis is more problematic because of the extended and clumped nature of chromosomes during this stage. Meiotic chromosomes become first identifiable in leptotene as fine long threads dispersed althrough nuclei (Fig. 1A). During zygotene homologous chromosomes begin to synapse along their length and appear as beaded structures (Fig. 1B). Subsequent sub-stages of prophase I nuclei corresponding to pachytene show shorter, thicker and more heavily stained beaded chromosome threads, with more conspicuous chiasmatic regions observable on long bivalents (Fig. 1C). Post-pachytene meiotic chromosomes exhibit clear crossover connections between homologues that severely constrain higher order axial compaction (Kleckner 1996). Meiotic studies, particularly of the late diplotene stage (Fig. 1E) have contributed towards establishing the chiasma frequency, averaged to 1.86 per bivalent and 13.1 per PMC. Chismata correspond to the points of physical exchange between non-sister chromatids of the homologues (Tease and Jones 1978) and their frequency is regarded as a good estimate of the level of genetic recombination in the species (Colombo 1992). In general, chiasmata are more frequent in euchromatin that contains actively transcribed genes than heterochromatin regions containing repeat sequences. Chiasmata analysis presents the primary source of information on meiotic recombination which gives rise to new combinations of alleles. At diplotene, homologues tend to be far apart owing to increased condensation except at the positions of chiasmata. In $A$. vera, bivalents are often not clearly visible due to clumping and stickiness of chromosomes even at diakinesis stage. This hurdle has been circumvented by preparation of a large number of adequately squashed slides increasing selection opportunities of well spread chromosomes. At diakinesis (Fig. 1F), seven bivalent consisting of four large and three small chromosome pairs were encountered. At metaphase I (Fig. 1G), extreme condensation of the bivalents makes them more prominent to discern. Despite clumping high success rate in chromosome pairing, chromosome disjunction at anaphase I and the distribution of chromatids at anaphase II later developing into microspores as also reported by Akinyele (2007) were noted in this species.

Meiotic chromosome aberrations In $A$. vera, meiosis is not always normal; earlier reports have also stated that several types of spontaneous chromosomal aberrations occur during microsporogenesis (Vig 1964; Sapre 1975; Imery 2007). At metaphase I stickiness, fragments and precocious movement of chromosomes were observed (Figs. 2A-C). According to Klasterskii et al. (1976) chromosome stickiness arises from improper folding of the chromosomes into single chromatids as a result of which chromatin fibers intermingle and chromosomes become attached to each other by means of sub-chromatid bridges. Gaulden (1987) postulated that sticky chromosomes may result from the defective functioning of one or two types of specific non-histone proteins involved in chromosome organization, which are needed for chromatid separation and segregation. In A. vera, the stickiness was intense and led to unequal separation of chromosomes. Anis and Wani (1997) have reported that precocious movement of chromosomes may be due to structural differentiation of homologous pair. Precocious 
Table 2. Karyomorhological features of Aloe vera

\begin{tabular}{|c|c|c|c|c|c|c|}
\hline $\begin{array}{l}\text { Chromosome } \\
\text { pair }\end{array}$ & $\begin{array}{l}\text { Short arm } \\
\qquad(\mu \mathrm{m})\end{array}$ & $\begin{array}{l}\text { Long arm } \\
\qquad(\mu \mathrm{m})\end{array}$ & $\begin{array}{c}\text { Total } \\
\text { chromosome } \\
\text { length }(\mu \mathrm{m})\end{array}$ & $\begin{array}{l}\text { Relative } \\
\text { length }\end{array}$ & $\begin{array}{c}\text { Arm ratio } \\
\mathrm{L} / \mathrm{S}\end{array}$ & $\begin{array}{l}\text { Centromere } \\
\text { type }\end{array}$ \\
\hline 1 & 4.00 & $10.37+2.23$ & 16.60 & 21.32 & 3.15 & St \\
\hline 2 & 3.75 & 11.50 & 15.25 & 19.59 & 3.07 & St \\
\hline 3 & 3.55 & 11.00 & 14.55 & 18.69 & 3.10 & St \\
\hline 4 & 2.81 & $6.84+2.05$ & 11.70 & 15.03 & 3.16 & $\mathrm{St}$ \\
\hline 5 & 2.50 & 4.60 & 7.10 & 9.12 & 1.84 & $\mathrm{Sm}$ \\
\hline 6 & 2.40 & 4.50 & 6.90 & 8.86 & 1.88 & $\mathrm{Sm}$ \\
\hline 7 & 2.00 & 3.75 & 5.75 & 7.39 & 1.88 & $\mathrm{Sm}$ \\
\hline
\end{tabular}

K.F. $2 n=2 x=14\left(4 \mathrm{St}^{\text {sat }}+4 \mathrm{St}+6 \mathrm{Sm}\right)$

chromosome migration to the poles can result into chaisma terminalization in diakinesis or metaphase I (Srivastava and Kapoor 2008). Of the chromosomal abnormalities, single bridge (4.8\%) recorded at anaphase I and II was found to be the most frequent one, which may be formed due to stickiness, unequal exchange or dicentric chromosomes (Fig. 2D). Sax (1960) and Saylor and Smith (1966) suggested that the formation of bridges might be due to the failure of chaismata to terminalise with the consequent effect that the bivalent chromosomes get interlocked and stretched between the poles. Double bridges also observed at anaphase I result from opposite orientation of the centromeres of dicentric chromosomes. A typical double bridge is shown in Fig. 2E formed by crisscross segregation of centromeres. Spontaneous breakage and fusion may have been responsible for bridge and fragment aberrations at anaphase. The laggards (Fig. 2F) have been attributed to the delayed terminalization, stickiness of chromosomal ends or failure of the chromosomal movement (Jayabalan and Rao 1987; Soheir et al. 1989). In most of the Aloe species bridges and chromosome fragments have been recorded between primary microsporocytes and the following mitotic anaphase/ telophase which are indicators of irregularities and deficiencies associated with gene duplication affecting the normal microsporogenesis and reduction in pollen fertility (Imery 2007). Though $87.60 \%$ pollen viability was recorded in A. vera, but surprisingly no seed setting was observed.

Karyomorphology of mitotic metaphase chromosomes Karyotype studies are regarded as an important cytogenetical tool for elucidating taxonomic and phylogenetic relationships vis a vis their usefulness in breeding programme aimed at developing superior genotypes (Stace 2000; Singh 1984). Aloaceae, one of the most stable angiosperm families for karyotypic orthoselection shows an extremely uniform chromosome constitution with $2 n=2 x=14$, comprised of eight long and six short chromosomes and shows a bimodal karyotype complement (Sapre 1978). Aloe vera chromosome complement is characterized by eight long subtelocentric chromosomes and six short submetacentric chromosomes (Figs. 3A and
3B). The idiogram (Fig. 4) substantiates the karyotypic formula (KF) $2 \mathrm{n}=14 \quad\left(4 \mathrm{St}^{\mathrm{sat}}+4 \mathrm{St}+6 \mathrm{Sm}\right)$. Within long chromosomes three pairs vary only slightly in their total length but fourth pair shows remarkable variation from others and the size of chromosomes within the complement ranges from 11.70 to $16.60 \mu \mathrm{m}$ in length. Vig (1968) has also reported the karyotype of this plant, and presented four long chromosomes in decreasing sizes of which the long chromosome pairs first and fourth possess satellites on their long arms having 2.23 and $2.05 \mu \mathrm{m}$ size, respectively. Three pairs of chromosomes are short and their size ranges from 5.75 to $7.10 \mu \mathrm{m}$ in length. According to Stebbins (1971) karyotype asymmetry of $A$. vera is 3B type. These findings support earlier observations of chromosome constitution for A. vera (Sapre 1978; Das et al. 2010; Gunjan and Roy 2010).

ACKNOWLEDGMENT. We gratefully acknowledge the financial assistance provided by the University Grant Commission, New Delhi, India.

\section{Literature Cited}

Akinyele, B. O. 2007. Meiotic chromosome behaviour and sexual sterility in two Nigerian species of Aloe Linn. Afr. Jour. Biotechnol 6: 2585-2589.

Alam, S. S. and Khanam, N. 2005. Fluorescent karyotype analysis of four Aloe species. Bangladesh Journ. Bot. 34: 17-20.

Anis, M. and Wani, A. A. 1997. Caffeine induced morphocytological variability in fenugreek, Trigonell foenumgraecum L. Cytologia 62: 343-349.

Anselm, A. 2004. Nature Power. 3rd ed. Fr. Anselm Adodo, OSB Ewu-Esan, Nigeria, p 288.

Colombo, P. C. 1992. A new index for estimating genetic recombination from chiasma distribution data. Heredity 69: 412-415.

Das, A., Mukherjee, P., Ghorai, A. and Jha, T. B. 2010. Comparative karyomorphological analyses of in vitro and in vivo grown plants of Aloe vera L. BURM. f. Nucleus 53: 89-94.

Fine, A. and Samuel, M. D. 1938. Cultivation and clinical application of Aloe vera leaf. Radiology 31: 735-736.

Gaulden, M. E. 1987. Hypothesis: some mutagens directly alter specific chromosomal proteins (DNA topoisomerase II and peripheral proteins) to produce chromosome stickiness, which causes chromosome aberrations. Mutagenesis 2: 357-365.

Grindlay, D. and Reynolds, T. 1986. The Aloe vera phenomenon: A review of the properties and modern use of leaf parenchyma gel. Journ. Ethno. Pharma. 16: 17-151. 
Gunjan, K. and Roy, B. K. 2010. Karyotype studies in dominant species of Aloe from eastern India. Caryologia 63: 41-49.

Heggers, J. P., Pelley, R. P. and Robson, M. C. 1993. Beneficial effects of Aloe in wound healing. Phytother. Res. 7: S48-S52.

Imery, J. 2007. Karyological instability during the formation of pollen mother cells in Aloe vera (Aloaceae). Int. J. Trop. Biol. 55: 805-813.

Jayabalan, N. and Rao, G. R. 1987. Gamma radiation induced cytological abnormalities in Lycopersicon esculentum Mill var. pusa ruby. Cytologia 52:1-4.

Klasterskii, I., Natarajan, A. T. and Ramel, C. 1976. An interpretation of the origin of subchromatid aberrations and chromosome stickiness as a category of chromatid aberrations. Hereditas 83:153.

Levan, A., Fredga, K. and Sandberg, A. A. 1964. Nomenclature for centromeric position on chromosomes. Hereditas 52: 201-220.

Kleckner, N. 1996. Meiosis: how could it work? Proc. Natl. Acad. Sci. USA 93:8167-8174.

Sapre, A. B. 1975. Meiosis and pollen mitosis in Aloe barbadensis Mill. (A. perfoliate var. vera L., A. vera Auth. non Mill.). Cytologia 40:525-533.

Sapre, A.B. 1978. Karyotype of Aloe barbadensis Mill.: A reinvestigation. Cytologia 43: 237-241.

Sax, K. 1960. Meiosis in Interspecific Pine hybrids Forest. Sci. 6: $135-138$.

Saylor, L. G. and Smith, B. N. 1966. Meiotic irregularities in species of iterspecific hybrids in Pisum. Am. Journ. Bot. 53: 453-468

Shelton, R. M. 1991. Aloe vera, its chemical and therapeutic properties. Int. Journ. Derm. 30: 679-683.

Sing, H. F. 1984. Pollen mitosis studies in orchids with particular reference to Cymbidium aloifolium SW. Current Sci. 53: 988-992.

Singh, J. P. and Dhuria, H. S. 1960. Studies on floral biology of sweet lime. Ind. Journ. Hort.17: 9-20.

Soheir, E. H. A. and Atif, H. 1989. Cytological effects of herbicide Garlon-4 on root mitosis of Allium cepa. Cytologia 54: 464-472.

Srivastava, A. and Kapoor, K. 1987. Seed yield is not impaired by chromosome stickiness in sodium azide treated Trigonella foenum-graecum. Cytologia 73: 115-121.

Stace, C. A. 2000. Cytology and cytogenetics as a fundamental taxonomic resource for the twenty and twenty first centuries. Taxon 49: 451-477.

Stebbins G. L. 1971. Chromosomal evolution in higher plants. Edward- Arnold (Publishers) Ltd., London, UK.

Tease, C. and Jones, G. H. 1978. Analysis of exchange in differential stained meiotic chromosome of Locusta migratoria BrdU-substitution and FPG staining: I. Crossover exchanges in monochiasmate bivalents. Chromosoma (Berl.) 69: 163-178.

Umesh, T. G. and Ranganath, R. M. 2003. Ultrautructural evidence for the flawless transmission of a dicentric chromosome in Aloe vera. L. Cytologia 68(3): 211-217.

Vig, B. K. 1968. Spontaneous chromosome abnormalities in roots and pollen mother cells in Aloe vera L. Bull. Torrey Bot. Club. 95: 254-261.

Winters, W. D., Benavides, R. and Clouse, W. J. 1981. Effects of Aloe extracts on human normal and tumor cells in vitro. Eco. Bot. 35: 89-95. 
